\title{
Detection of Bacillus anthracis and Bacillus anthracis-like spores in soil from state of Rio de Janeiro, Brazil
}

\author{
Jacqueline RS Salgado'/ ${ }^{+}$, Leon Rabinovitch ${ }^{2}$, Maria de Fátima dos S Gomes', \\ Regina Celia da SB Allit ${ }^{3}$, Marcelo Martins Werneck ${ }^{3}$, Rafael B Rodrigues', \\ Renata C Picão ${ }^{4}$, Fernanda Baptista de Oliveira Luiz², Adriana M Vivoni
}

${ }^{1}$ Exército Brasileiro, Instituto de Defesa Química, Biológica, Radiológica e Nuclear, Laboratório de Defesa Biológica, Rio de Janeiro, RJ, Brasil ${ }^{2}$ Fundação Oswaldo Cruz-Fiocruz, Instituto Oswaldo Cruz, Laboratório de Fisiologia Bacteriana/Laboratório de Referência Nacional para Carbúnculo, Rio de Janeiro, RJ, Brasil

${ }^{3}$ Universidade Federal do Rio de Janeiro, Instituto Alberto Luiz Coimbra de Pós-Graduação e Pesquisa de Engenharia,

Laboratório de Instrumentação e Fotônica, Rio de Janeiro, RJ, Brasil

${ }^{4}$ Universidade Federal do Rio de Janeiro, Instituto de Microbiologia Paulo de Góes, Rio de Janeiro, RJ, Brasil

BACKGROUND Bacillus anthracis is the aetiologic agent of anthrax, a re-emerging, septicaemic, haemorrhagic and lethal disease that affects humans, domestic ruminants and wildlife. Plasmids pXO1 and pXO2 are attributes that confer pathogenicity to $B$. anthracis strains. This bacterium was used as biological weapon in the World Wars and in the biological attack in the United States of America at 2001. B. anthracis is classified as a Tier 1 bioterrorism agent by the Centers for Diseases Control and Prevention. Anthrax is recognised as a re-emerging disease. Several studies concerning the dynamics of B. anthracis cycle in soil revealed that nonpathogenic $B$. anthracis strains due to lack of $\mathrm{pXO} 2$ plasmid are commonly found in some types of soil.

OBJECTIVES This study aimed isolation and identification of $B$. anthracis spores in soil samples of the state of Rio de Janeiro, Brazil.

METHODS Phenotypic and genotypic approaches were used to identify isolates including MALDI-TOF/MS, motility test, susceptibility to gamma phage and penicillin, survey for pag and cap genes as surrogates of pXO1 and pXO2 plasmids, respectively, and sequencing of 16SrRNA-encoding gene. Physicochemical analysis of the soil samples were carried out to describe soil characteristics.

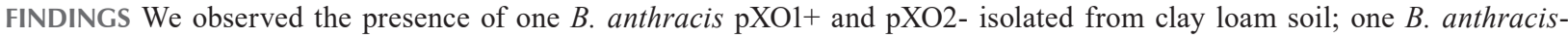
like strain pXO1+ and pXO2-isolated from loamy sand; and 10 Bacillus spp. strains sensitive to phage-gamma that need better characterisation to define which their species were recovered from loamy sand.

MAIN CONCLUSIONS This work showed promising results and it was the first study to report results from an active surveillance for B. anthracis in Brazil.

Key words: spores - Bacillus anthracis- Bacillus anthracis-like - anthrax and soil

Bacillus anthracis is a non-motile, non-haemolytic, aerobic Gram-positive endospore-forming rod. It belongs to the $B$. cereus group, which comprises at least eight closely related species: $B$. anthracis, $B$. cereus, $B$. thuringiensis, $B$. mycoides, $B$. pseudomycoides, $B$. weihenstephanensis, B. cytotoxicus and B. Toyonensis. ${ }^{(1)} B$. anthracis pathogenicity is mainly due to its ability to produce toxins and capsule, which are encoded by pXO1 and pXO2 plasmids, respectively. It is the aetiologic agent of anthrax, a zoonotic, septic, haemorrhagic and lethal disease that affects mostly domestic and wild ruminants. ${ }^{(2)}$ B. anthracis is ubiquitous in nature and the spores are resistant to drying, radiation and disinfectants.

doi: 10.1590/0074-02760200370

Financial support: IDQBRN, COPPE/UFRJ, LFB/IOC/FIOCRUZ, IMPG/

UFRJ. All institutions had the material to carry out this research.

+ Corresponding author: capjrsoares@gmail.com

(D) https://orcid.org/0000-0002-4664-9222

Received 12 July 2020

Accepted 14 October 2020
B. anthracis was used in bioweapon programs of many countries, such as Germany during World War I, Japan in World War II, the former Union of Soviet Socialist Republics (USSR) (1928-1992), United States of America (USA) (1941-1969), Iraq (1970-1991) and others. In addition, it was used in bioterrorist attacks perpetrated by Aum Shinrikyo (1995, Japan, no fatalities) and the Amerithrax (2001, USA, five people killed and 22 infected). It is classified by the Centers for Disease Control and Prevention (CDC) as a Tier 1 biological agent. ${ }^{(3,4)}$

It can be isolated from environmental sources such as soil and water, and from food products. B. anthracis spores can remain viable for years in soils with $\mathrm{pH}$ between 6 and 8.5, especially at the deeper layers. Changes such as plowing or drainage, however, carry them to the surface. Outbreaks of anthrax are frequent in tropical and subtropical countries with high annual rainfalls and are common after major changes in weather, such as heavy rains after a long period of drought, or a dry summer after heavy rains, always in temperatures above $15^{\circ} \mathrm{C}$. Soil conditions such as $\mathrm{pH}$, salts, organic matter, temperature, humidity and microbial burden vary according climatic seasons. ${ }^{(5)}$ Regardless of how $B$. anthracis spores reach the ground, it is generally accepted that 
some soils are more likely to harbour spores than others. B. anthracis is most frequently found in clayey soils rich in organic matter and $\mathrm{Ca}^{2+}$, with $\mathrm{pH}$ above 6.0 and temperatures above $15.5^{\circ} \mathrm{C} .(6,7)$

According to Schild et al., ${ }^{(8)}$ the disease occurs all over South America where Brazil share borders (total length of $16,885.7 \mathrm{~km}$ ) with 10 out of 12 countries. From 2006 until July 2019, Argentina (borders with Rio Grande do Sul, Santa Catarina e Paraná states) reported 144 anthrax outbreaks, Uruguay (borders with Rio Grande do Sul state) 63 outbreaks, Paraguay (borders with Paraná e Mato Grosso do Sul states) 54 outbreaks, Peru (borders with Acre e Amazonas states)18 outbreaks, Bolivia (borders with Mato Grosso e Rondônia states) 38 outbreaks and Colombia (border with Amazonas state) 11 outbreaks..$^{(9)}$ Brazil's vast border extension can make it vulnerable to the clandestine entry of contaminated animals.

In Brazil, anthrax is on the list of diseases that requires animal health protection measures since 1934, requiring the sacrifice of affected animals and mandatory notification. According to the Ministry of Agriculture, Livestock and Supply, the last reported animal case occurred in 2016, in the state of Rio de Janeiro. ${ }^{(10)}$ Regarding to human disease, nine cases of cutaneous anthrax were reported, between 1930-1932, all due to contact with contaminated animals..$^{(1)}$

Since 1928, anthrax in cattle and goats mostly occurs in three distinct areas of Brazil: south and west region of Rio Grande do Sul, where some places were called "cursed fields"; Paraiba river valley in São Paulo and Minas Gerais states; and in the northeast region. Among 72 outbreaks of sudden death in cattle that occurred between 2000 and 2014 in Rio Grande do Sul, seven were identified as caused by B. anthracis. ${ }^{(12)}$ Ten outbreaks of anthrax were confirmed from January 1978 to March 2006 in Brazil occurred in cattle, most non-vaccinated, in the southeastern and southern region of Rio Grande do Sul, in municipalities on the Uruguay border. Lack of vaccination may have been an adjuvant for the occurrence of the disease after exposure to a primary source, such as soil from an old anthrax grave. ${ }^{(8)}$

Several studies on the dynamics of $B$. anthracis cycle in soil have been carried out. ${ }^{(7,13)}$ However, there is no data available about the occurrence of $B$. anthracis in soil in Brazil and very little information about Brazilian strains. The aim of this research was to survey $B$. anthracis in soil samples from Brazil, correlated or not with anthrax cases.

\section{MATERIALS AND METHODS}

Soil samples - Soil samples were collected at two different geographical sites in Rio de Janeiro state: in Barra de Guaratiba [three soil samples, two from $\mathrm{Fa}$ zenda Modelo and one from Army Technology Center (CTEx)], sites not correlated with anthrax cases and in Barra do Piraí, location of the last case of anthrax reported in Brazil, in 2016, a cattle burrier site. Table I shows geographical coordinates of sampling.

Soil samples $(20 \mathrm{~g})$ were collected with sterile stainless-steel spatulas at 2 to $5 \mathrm{~cm}$ depth. Sampling areas with $50 \mathrm{~m}^{2}$ were delimited and subsamples were col- lected at the angles (A, B, C and D) and at the central point (E), totalising $100 \mathrm{~g}$ in each collection site (Figure). These samples were stored at $4^{\circ} \mathrm{C}$ until processing.

Physicochemical analysis of soil samples - Physicochemical characterisation of soil samples was performed at the Analytical Centre Laboratory of the Federal Rural University of Rio de Janeiro (UFRRJ). The main features for $B$. anthracis spore persistence in soil, such as granulometry, $\mathrm{Ca}^{2+}$ concentration, $\mathrm{pH}$ and organic matter content were analysed. Values suggested by the Brazilian Agricultural Research Corporation (EMBRAPA) and the Commission of Soil Fertility of Minas Gerais State (CFSEMG) were used for comparison. ${ }^{(14,15)}$ The definition of soils textural class was based on the Triangle Diagram from the United States Department of Agriculture (USDA). ${ }^{(16)}$

Soil processing - All samples were submitted to heat treatment at $70^{\circ} \mathrm{C}$ for $15 \mathrm{~min}$ in water bath. Serial dilutions were performed in SATAMP up to $10^{-9}$. From each serial dilution, $100 \mu \mathrm{L}$ were transferred and plated with Digralski loops in the culture media and incubated at $33^{\circ} \mathrm{C}$ for $24 \mathrm{~h}$. Culture media used in this study were: nutrient agar (NA) (Difco ${ }^{\mathrm{TM}}$ ), NA with $0.25 \mathrm{M}$ sodium acetate $(\mathrm{NA}+)\left(\mathrm{Difco}^{\mathrm{TM}}\right)$, Columbia agar base $\left(\mathrm{Difco}^{\mathrm{TM}}\right)$ with $5 \%$ of sheep blood (CBA), CBA with $0.25 \mathrm{M}$ sodium acetate $(\mathrm{CBA}+), \mathrm{CBA}$ with $0.5 \%$ sodium bicarbonate $(\mathrm{CBA}++)$ and PLET agar (Sigma-Aldrich $-15 \mathrm{~g} / \mathrm{L}$ agar, $30,000 \mathrm{U} / \mathrm{L}$ polymyxin $\mathrm{b}, 300,000 \mathrm{U} / \mathrm{L}$ lysozyme, 300 $\mathrm{mg} / \mathrm{L}$ EDTA and $40 \mathrm{mg} / \mathrm{L}$ thallium acetate). Samples

\section{TABLE I}

Geographic coordinates of soil sampling

\begin{tabular}{lcc}
\hline Code $^{a}$ & Location & $\begin{array}{c}\text { Geographic coordinates } \\
\text { (latitude/longitude) }^{b}\end{array}$ \\
\hline BP1 & Barra do Piraí & $22.41433 / 43.56128$ \\
\hline BP2 & Barra do Piraí & $22.41429 / 43.56114$ \\
\hline BP3 & Barra do Piraí & $22.41440 / 43.56129$ \\
\hline CT & Barra de Guaratiba & $23.030097 / 43.575808$ \\
\hline CA & Barra de Guaratiba & $22.993756 / 44.590284$ \\
\hline CB & Barra de Guaratiba & $22.994050 / 43.592963$
\end{tabular}

$a$ : BP1 - cattle burier site; $b$ : coordinates obtained from Google Maps $^{\circledR}$. BP2: site of death of a contaminated bovine $(24 \mathrm{~h})$; BP3: grass covered soil; CA: corral A soil; CB: corral B soil; CT: Army Technology Center (CTEx) soil.

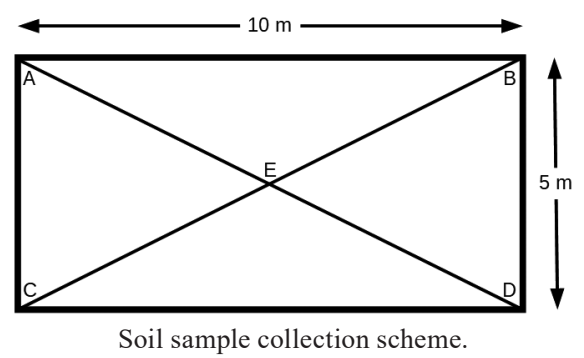


were homogenised with manual rotational motion for $30 \mathrm{~min}$. Subsequently, $1 \mathrm{~g}$ of soil was transferred to 100 $\mathrm{mL}$ of sterile buffered saline $(0,2 \mathrm{M}$ potassium hydrogen phosphate - SATAMP - pH 7.2) and homogenised at $33^{\circ} \mathrm{C}$ for $30 \mathrm{~min}$ at $150 \mathrm{rpm}$ (Shaker Innova 4080 , New Brunswick Scientific Co., USA).

Colonies presenting characteristic $B$. anthracis morphology were selected and submitted to microscopic examination using Gram staining. Gram-positive bacteria presented suggestive characteristics of $B$. anthracis ("box-shapped" cells with 1-1.5 $\mu \mathrm{m}$ width and 4-10 $\mu \mathrm{m}$ length and subterminal, cylindrical and non-deforming spores) were further characterised as described below.

Identification of isolates suspected of B. anthracis Isolates presenting $B$ anthracis characteristic colonies and cell morphology were further evaluated for gamma phage and penicillin susceptibility, motility and presence of pag and cap genes by specific PCR and MALDI-TOF/MS (Microflex LT mass spectrometer, Bruker, USA) as described below.

B. anthracis (CCGB 1861) was used as positive control and B. cereus (CCGB 0406 LFB-Fiocruz 406) as negative control. Both strains were provided by Collection of Genus Bacillus and Related Genera-CCGB-Fiocruz.

The strains received a numeral code for identification (Table II). The results of the 52 isolates are shown in Table II.

Motility test - To evaluate motility a $1 \mu \mathrm{L}$ bacterial loop of each isolate was inoculated in $5 \mathrm{ml}$ of nutrient broth with $0.5 \%$ of $\mathrm{D}-(+)$-glucose and incubated in anaerobic conditions at $33^{\circ} \mathrm{C}$ for $20 \mathrm{~h}$. Motility was observed on a fresh exam of broth culture by optical microscopy (x1000) (NiKon, Japan).

Susceptibility to gamma phage and penicillin - For the gamma phage susceptibility assay, $1 \mu \mathrm{L}$ of $7.9 \times 10^{9}$ $\mathrm{PFU} / \mathrm{mL}$ gamma phage suspension [provided by Public Health Agency (HPA), Porton Down, England] was used. For the penicillin susceptibility test, 10 U.I. Sensibiodisc (CECON, Brazil) were used. Samples grown in $\mathrm{CBA}$ were inoculated in nutrient broth and incubated at $33^{\circ} \mathrm{C}$ for $24 \mathrm{~h}$. Bacterial suspensions were inoculated into CBA plates using sterile swab. After the inoculation, penicillin disks and a $10-\mu \mathrm{L}$ drop of gamma phage suspension were placed on the surface and plates were incubated at $37^{\circ} \mathrm{C}$ for $24 \mathrm{~h}$ in aerobic conditions. Absence of bacterial growth as lysis plates and inhibition zones around the penicillin disk of any size were considered as positive results.

Microbial identification by MALDI-TOF/MS - Mass spectra were acquired using a Microflex LT mass spectrometer and results were analysed using Biotyper software version 3.1 (Bruker Daltonics, USA) containing a bioterrorism biological agent library, including 23 virulent $B$. anthracis strains and the Sterne strain.

Sample inactivation was performed according to Lasch et al. ${ }^{(17)}$ Briefly, $1 \mu \mathrm{L}$ of each sample was transferred to a 96-well stainless-steel plate and allowed to dry at room temperature. Then, $1 \mu \mathrm{L}$ of $\alpha$-cyano-4hydroxycinnamic acid matrix (HCCA) was added and allowed to dry at room temperature. MALDI-TOF MS analysis was performed in duplicate by linear mode with the following parameters: laser frequency $20 \mathrm{~Hz}$; voltage of ion sources 1 and $2,20 \mathrm{kV}$ and $18.6 \mathrm{kV}$, respectively; molecular weight range 2,000 to 20,000 Da. Mass spectra were collected through the sum of 240 laser shots, acquired through four groups of 60 shots cast in randomly selected distinct regions. The spectra of the samples were calibrated internally, using Escherichia coli DH5 $\alpha$ (IDQBRN, Brazil) ribosomal proteins as reference. Spectra were processed by Biotyper software in standard operating mode, which performs the research in its reference library in order to get the best match for the sample. Results were scored ranging from 0 to 3.0. Scores below 1.7 were considered unreliable, those between 1.7 and 2.0 were considered possible indicator of genus, those between 2.0 and 2.3 were considered reliable for genus and possible for species identification and those above 2.3 were considered reliable for identification at the species level.

Evaluation of pag and cap genes in isolates suspected of $B$. anthracis - To survey the presence of pag and cap genes, a multiplex PCR was performed using Beyer 8 (TCC-TAA-CAC-TAA-CGA-AGT-CG), Beyer 5 (GAG-GTA-GAA-GGA-TAT-ACG-GT), 1234 (CTGAGC-CAT-TAA-TCG-ATA-TG) and 1301 (TCC-CACTTA-CGT-AAT-CTG-AG) primers. ${ }^{(3,18)}$

DNA extraction was carried out using Instagene matrix kit (Bio-Rad, USA) according to the manufacturer's instructions. PCR reactions were performed as previously described ${ }^{(17,18)}$ Briefly, multiplex PCR reactions for pag and cap were performed in $25 \mu \mathrm{L}$ reaction volume containing $2.5 \mu \mathrm{L}$ of template DNA, $2.5 \mu \mathrm{L}$ of $10 \times$ PCR Buffer, $0.5 \mu \mathrm{L}$ of DNTP, $1,25 \mu \mathrm{L}$ of a $10 \mu \mathrm{M}$ solution of each primer, $14.38 \mu \mathrm{L}$ of DNAse free water (Thermo Fisher Scientific, USA) and $0,0125 \mu \mathrm{L}$ of Taq. For amplification, the following parameters were used: an initial denaturation step of $95^{\circ} \mathrm{C}$ for $5 \mathrm{~min} ; 30$ cycles of $95^{\circ} \mathrm{C}$ for $1 \mathrm{~min} ; 55^{\circ} \mathrm{C}$ for $30 \mathrm{sec} ; 72^{\circ} \mathrm{C}$ for $30 \mathrm{sec}$; and a final extension step at $72^{\circ} \mathrm{C}$ for $5 \mathrm{~min} . .^{(19,20)}$

PCR products were observed after $1 \%$ agarose gel electrophoresis containing ethidium bromide under ultraviolet light.

PCR amplicon sequencing - Isolates positive for pag genes were submitted to simplex PCR, as described above, with the pag primers. PCR products were purified using Exosap-IT Express (Thermo Fisher Scientific, USA), according to the manufacture instructions. Amplicons were sequenced in an ABI3730XL automated sequencer (Applied Biosystems). Forward and reverse sequences were aligned and edited using Seqman program (DNAstar, Larsegene, version 7.0) and the data compared to the sequences deposited in GenBank with nBLAST.

16S rRNA gene sequencing - Isolates positive for pag genes and those susceptible to gamma phage were submitted to amplification and sequencing of the gene encoding the $16 \mathrm{~S}$ rRNA, according to Watts et al., ${ }^{(21)}$ with universal primers pA (5'-AGA-GTT-TGA-TCC-TGG-CTC-AG), pH (5'-AAG-GAG-GTG-ATC-CAG-CCG-CA), 1831 (5'-GAG-GAA-CAC-CGA-TGG-CGA-AGG-C) and 1832 (5'-GCC-CCC-GTC-AAT-TCC-TTT-GAG-TT). 
Products were purified using Exosap-IT Express (Thermo Fisher Scientific, USA), according to the manufacture instructions and sequenced at the DNA Capillary sequencing Facility - SANGER (Fiocruz, Brazil) in an ABI3730XL automated sequencer (Applied Biosystems). Forward and reverse sequences were aligned and edited using BioEdit 7.2 software and data were compared to the sequences in GenBank using nBLAST. The sequences were submitted at GenBank.

\section{RESULTS}

Physicochemical characteristics of soil samples The textural class of all samples was identified after particle size analysis. Samples BP1 and BP2 were classified as Loam: BP3 as Sandy Clay Loam, CA as Clay loam; $\mathrm{CB}$ and CT as Sandy Loam (Table III).

Regarding $\mathrm{pH}$ analysis, BP1 presented weak acidity while the other samples presented medium acidity.

TABLE II

Results of 52 isolates

\begin{tabular}{|c|c|c|c|c|c|c|c|c|c|c|c|c|}
\hline 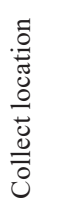 & 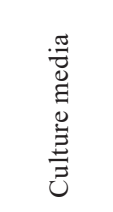 & $\frac{\tilde{\theta}}{0}$ & 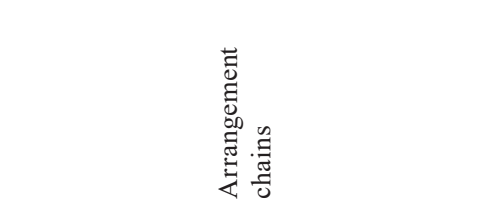 & 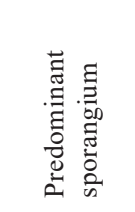 & 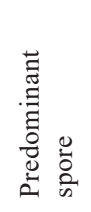 & 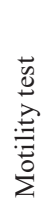 & 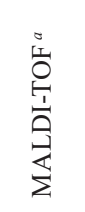 & 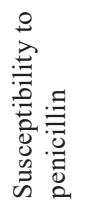 & 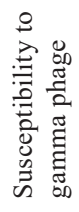 & 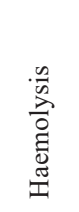 & 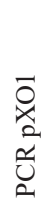 & 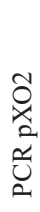 \\
\hline \multirow{10}{*}{ CT } & \multirow{3}{*}{ CBA } & 8 & Short & St, Nd, Ci & Ws & - & $\begin{array}{c}1.636 \\
\text { NI }\end{array}$ & $\mathrm{R}$ & $\mathrm{S}$ & A & - & - \\
\hline & & 32 & Long, isolated cells and in pairs & St, d, Ci & $\mathrm{Ci}$ & - & $\begin{array}{c}1.959 \\
\mathrm{Ba}\end{array}$ & $\mathrm{R}$ & $\mathrm{S}$ & A & - & - \\
\hline & & 40 & Long & St, Nd, Ci & $\mathrm{Ci}$ & - & $\begin{array}{c}1.988 \\
\mathrm{Ba}\end{array}$ & S & $\mathrm{R}$ & A & - & - \\
\hline & $\mathrm{CBA}++$ & 2 & Short & Ws & $\mathrm{Ci}$ & - & $\begin{array}{c}1.949 \\
\mathrm{Ba}\end{array}$ & $\mathrm{R}$ & $\mathrm{S}$ & A & - & - \\
\hline & \multirow{3}{*}{$\mathrm{CBA}+$} & 3 & Long & St, Nd, Ci & $\mathrm{Ci}$ & - & $\begin{array}{c}2.016 \\
\mathrm{Ba}\end{array}$ & $\mathrm{R}$ & $\mathrm{S}$ & A & - & - \\
\hline & & 5 & Long and short & St, Nd, Ci & Ws & - & $\begin{array}{c}2.078 \\
\mathrm{Ba}\end{array}$ & $\mathrm{R}$ & $\mathrm{R}$ & A & - & - \\
\hline & & 10 & Long, isolated cells and in pairs & St, Nd, Ci & Ws & - & $\begin{array}{c}1.424 \\
\text { NI }\end{array}$ & S & $\mathrm{R}$ & Beta & - & - \\
\hline & \multirow{3}{*}{ NA } & 4 & Long & St, Nd, Ci & $\mathrm{Ci}$ & - & $\begin{array}{c}2.065 \\
\mathrm{Ba}\end{array}$ & $\mathrm{R}$ & $\mathrm{S}$ & A & - & - \\
\hline & & 33 & Short & St, Nd, Ci & Ws & - & $\begin{array}{c}1.948 \\
\mathrm{Ba}\end{array}$ & S & $\mathrm{R}$ & Beta & - & - \\
\hline & & 9 & Long & St, Nd, Ci & $\mathrm{Ci}$ & - & $\begin{array}{c}2.000 \\
\mathrm{Ba}\end{array}$ & $\mathrm{R}$ & $\mathrm{R}$ & A & - & - \\
\hline \multirow{11}{*}{ CT } & NA & 41 & Long & $\mathrm{St}, \mathrm{Nd}, \mathrm{Ci}$ & $\mathrm{Ci}$ & + & $\begin{array}{c}1.856 \\
\mathrm{Ba}\end{array}$ & $\mathrm{R}$ & $\mathrm{R}$ & Beta & + & - \\
\hline & \multirow{2}{*}{ NA+ } & 20 & Long & Ws & Ws & - & $\begin{array}{c}1.891 \\
\mathrm{Ba}\end{array}$ & S & $\mathrm{R}$ & Beta & - & - \\
\hline & & 21 & Long & $\mathrm{St}, \mathrm{Nd}, \mathrm{Ci}$ & Ws & - & $\begin{array}{c}1.906 \\
\mathrm{Ba}\end{array}$ & $\mathrm{R}$ & $\mathrm{R}$ & A & - & - \\
\hline & \multirow{8}{*}{ PLET } & 22 & Short & St, Nd, E & Ws & - & $\begin{array}{c}1.861 \\
\mathrm{Ba}\end{array}$ & $\mathrm{R}$ & $\mathrm{R}$ & Alfa & - & - \\
\hline & & 24 & Short & St, Nd, Ci & Ws & - & $\begin{array}{c}1.922 \\
\mathrm{Ba}\end{array}$ & S & $\mathrm{R}$ & A & - & - \\
\hline & & 34 & Short & St, Nd, Ci & $\mathrm{Ci}$ & - & $\begin{array}{c}1.608 \\
\text { NI }\end{array}$ & S & $\mathrm{R}$ & A & - & - \\
\hline & & 36 & Short & St, Nd, Ci & Ws & - & $\begin{array}{c}1.817 \\
\mathrm{Ba}\end{array}$ & $\mathrm{R}$ & $\mathrm{S}$ & A & - & - \\
\hline & & 37 & Isolated cells and in pairs & $\mathrm{St}, \mathrm{Nd}, \mathrm{Ci}$ & Ws & - & $\begin{array}{c}1.672 \\
\text { NI }\end{array}$ & $\mathrm{R}$ & $\mathrm{S}$ & A & - & - \\
\hline & & 31 & Long in curve, isolated cells and in pairs & St, Nd, Ci & $\mathrm{Ci}$ & - & $\begin{array}{c}2.006 \\
\mathrm{Ba}\end{array}$ & $\mathrm{S}$ & $\mathrm{R}$ & A & - & - \\
\hline & & 39 & Short & St, Nd, Ci & Ws & + & $\begin{array}{c}1.969 \\
\mathrm{Ba}\end{array}$ & R. & $\mathrm{R}$ & Alfa & - & - \\
\hline & & 42 & Short & St, Nd, Ci & $\mathrm{Ci}$ & - & $\begin{array}{c}1.700 \\
\mathrm{Ba}\end{array}$ & $\mathrm{S}$ & $\mathrm{R}$ & A & - & - \\
\hline
\end{tabular}




\begin{tabular}{|c|c|c|c|c|c|c|c|c|c|c|c|c|}
\hline 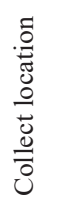 & 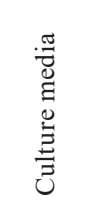 & $\begin{array}{l}0 \\
0 \\
0\end{array}$ & 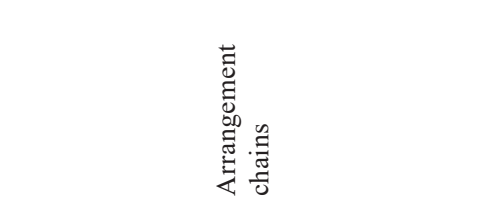 & 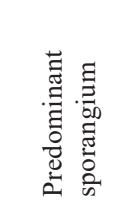 & 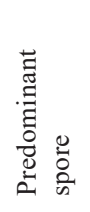 & 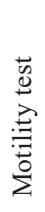 & 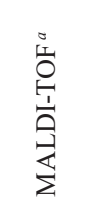 & 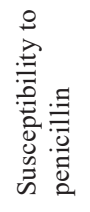 & 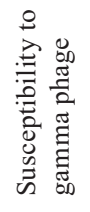 & $\begin{array}{l}\frac{n}{\tilde{n}} \\
\frac{\partial}{0} \\
\frac{\Xi}{0} \\
\tilde{\Xi}\end{array}$ & $\begin{array}{l}\overline{0} \\
\text { a } \\
\text { ô } \\
\text { }\end{array}$ & 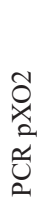 \\
\hline $\mathrm{CB}$ & NA+ & 18 & Long & St, Nd, E & Ws & + & $\begin{array}{c}2.068 \\
\mathrm{Ba}\end{array}$ & $\mathrm{S}$ & $\mathrm{R}$ & A & - & - \\
\hline \multirow{11}{*}{ CA } & \multirow{4}{*}{ NA } & 6 & Long, isolated cells and in pairs & St, Nd, Ci & Ws & - & $\begin{array}{c}1.832 \\
\mathrm{Ba}\end{array}$ & $\mathrm{R}$ & $\mathrm{S}$ & A & - & - \\
\hline & & 11 & Long & St, Nd, Ci & $\mathrm{Ci}$ & + & $\begin{array}{c}1.435 \\
\mathrm{NI}\end{array}$ & $\mathrm{S}$ & $\mathrm{R}$ & A & - & - \\
\hline & & 17 & Long, isolated cells and in pair & St, Nd, Ci & $\mathrm{Ci}$ & - & $\begin{array}{c}1.761 \\
\mathrm{Ba}\end{array}$ & S & $\mathrm{R}$ & A & - & - \\
\hline & & 38 & Long and short & St, Nd, Ci & Ws & - & $\begin{array}{c}2.045 \\
\mathrm{Ba}\end{array}$ & $\mathrm{S}$ & $\mathrm{R}$ & A & - & - \\
\hline & \multirow{7}{*}{ PLET } & 7 & $\begin{array}{l}\text { Short, isolated cells in pairs, } \\
\text { suggesting poles with right angles }\end{array}$ & St, Nd, Ci & $\mathrm{Ci}$ & - & $\begin{array}{c}1.941 \\
\mathrm{Ba}\end{array}$ & $\mathrm{R}$ & $\mathrm{S}$ & A & - & - \\
\hline & & 23 & Long & St, Nd, Ci & $\mathrm{Ci}$ & - & $\begin{array}{c}1.365 \\
\mathrm{NI}\end{array}$ & $\mathrm{R}$ & $\mathrm{R}$ & Alfa- & - & - \\
\hline & & 25 & Curved shorts, isolated cells and in pairs & St, Nd, Ci & Ws & - & $\begin{array}{c}1.903 \\
\mathrm{Ba}\end{array}$ & $\mathrm{S}$ & $\mathrm{R}$ & A & - & - \\
\hline & & 26 & Curved shorts, isolated cells and in pair & St, Nd, Ci & $\mathrm{Ci}$ & - & $\begin{array}{c}1.729 \\
\mathrm{Bp}\end{array}$ & $\mathrm{S}$ & $\mathrm{R}$ & A & - & - \\
\hline & & 28 & Shorts, isolated cells and in pair & St, Ld, Ci & $\mathrm{Ci}$ & - & $\begin{array}{c}1.988 \\
\mathrm{Ba}\end{array}$ & $\mathrm{S}$ & $\mathrm{R}$ & A & - & - \\
\hline & & 29 & $\begin{array}{l}\text { Short, isolated cells in pairs, } \\
\text { suggesting a right-angle pole }\end{array}$ & St, Nd, Ci & $\mathrm{Ci}$ & - & $\begin{array}{c}2.315 \\
\mathrm{Ba}\end{array}$ & $\mathrm{S}$ & $\mathrm{S}$ & A & + & - \\
\hline & & 30 & $\begin{array}{l}\text { Short, isolated cells in pairs, } \\
\text { suggesting a right-angle pole }\end{array}$ & St, Nd, Ci & Ws & - & $\begin{array}{c}1.944 \\
\mathrm{Ba}\end{array}$ & $\mathrm{R}$ & $\mathrm{S}$ & A & - & - \\
\hline \multirow{12}{*}{ BP1 } & CBA & 51 & Long & St, Nd, Ci & $\mathrm{Ci}$ & - & $\begin{array}{c}2.043 \\
\mathrm{Bm}\end{array}$ & S & $\mathrm{R}$ & A & - & - \\
\hline & \multirow{3}{*}{ CBA+ } & 55 & Long & St, Nd, Ci & $\mathrm{E}$ & - & $\begin{array}{c}1.73 \\
\mathrm{Ba}\end{array}$ & S & $\mathrm{R}$ & Beta & - & - \\
\hline & & 66 & $\begin{array}{l}\text { Long, isolated cells in pairs, } \\
\text { suggesting poles with right angles }\end{array}$ & St, Nd, Ci & $\mathrm{Ci}$ & + & $\begin{array}{c}1.518 \\
\mathrm{NI}\end{array}$ & $\mathrm{R}$ & $\mathrm{R}$ & A & - & - \\
\hline & & 84 & Long, isolated cells in pairs & St, Nd, Ci & $\mathrm{Ci}$ & - & $\begin{array}{c}1.634 \\
\mathrm{NI}\end{array}$ & $\mathrm{R}$ & $\mathrm{R}$ & A & - & - \\
\hline & \multirow{8}{*}{ PLET } & 69 & Short & St, Nd, Ci & Ws & - & $\begin{array}{c}1.417 \\
\mathrm{NI}\end{array}$ & $\mathrm{R}$ & $\mathrm{R}$ & A & - & - \\
\hline & & 72 & Short & St, Nd, Ci & Ws & + & $\begin{array}{c}2.022 \\
\mathrm{Ba}\end{array}$ & $\mathrm{R}$ & $\mathrm{R}$ & Beta & - & - \\
\hline & & 73 & Short, cells in pairs & St, Nd, E & Ws & - & $\begin{array}{c}1.563 \\
\mathrm{NI}\end{array}$ & $\mathrm{R}$ & $\mathrm{R}$ & A & - & - \\
\hline & & 81 & Short, isolated cells and in pairs & St, Nd, Ci & $\mathrm{E}$ & + & $\begin{array}{c}2.212 \\
\mathrm{Bl}\end{array}$ & $\mathrm{R}$ & $\mathrm{R}$ & A & - & - \\
\hline & & 82 & Short, isolated cells and in pairs & St, Nd, Ci & $\mathrm{Ci}$ & - & $\begin{array}{c}1.619 \\
\mathrm{NI}\end{array}$ & $\mathrm{R}$ & $\mathrm{R}$ & A & - & - \\
\hline & & 90 & Short, isolated cells and in pairs & $\mathrm{St}, \mathrm{Nd}, \mathrm{E}$ & Ws & + & $\begin{array}{l}1.308 \\
\mathrm{NI}\end{array}$ & $\mathrm{R}$ & $\mathrm{R}$ & A & - & - \\
\hline & & 93 & Short, isolated cells and in pairs & St, Nd, Ci & Ws & + & $\begin{array}{l}1.204 \\
\mathrm{NI}\end{array}$ & $\mathrm{R}$ & $\mathrm{R}$ & Beta & - & - \\
\hline & & 97 & Short, isolated cells & St, Nd, Ci & $\mathrm{Ci}$ & + & $\begin{array}{c}1.428 \\
\mathrm{NI}\end{array}$ & $\mathrm{S}$ & $\mathrm{R}$ & A & - & - \\
\hline
\end{tabular}




\begin{tabular}{|c|c|c|c|c|c|c|c|c|c|c|c|c|}
\hline 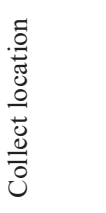 & 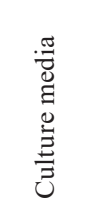 & $\begin{array}{l}\tilde{d} \\
0 \\
0\end{array}$ & 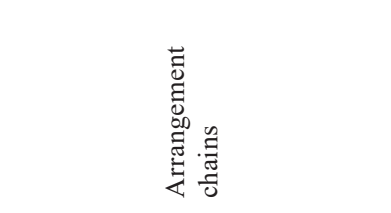 & 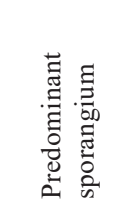 & 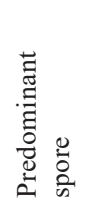 & 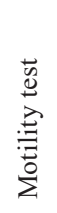 & 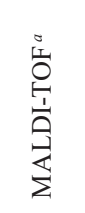 & 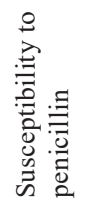 & 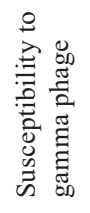 & $\begin{array}{l}\frac{n}{0} \\
\frac{n}{0} \\
\stackrel{0}{0} \\
\frac{\pi}{ \pm}\end{array}$ & 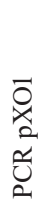 & 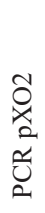 \\
\hline \multirow{4}{*}{ BP2 } & \multirow{4}{*}{ PLET } & 49 & Short & St, Nd, Ci & $\mathrm{Ci}$ & - & $\begin{array}{c}2.073 \\
\mathrm{Ba}\end{array}$ & $\mathrm{R}$ & $\mathrm{R}$ & Beta & - & - \\
\hline & & 53 & Short, isolated cells and in pairs & St, Nd, Ci & $\mathrm{E}$ & - & $\begin{array}{c}1.853 \\
\mathrm{Ba}\end{array}$ & $\mathrm{R}$ & $\mathrm{R}$ & Beta & - & - \\
\hline & & 59 & Short & St, Nd, Ci & E & + & $\begin{array}{l}1.335 \\
\mathrm{NI}\end{array}$ & $\mathrm{R}$ & $\mathrm{R}$ & Beta & - & - \\
\hline & & 78 & Short, cells in pairs & St, Nd, Ci & $\mathrm{Ci}$ & + & $\begin{array}{c}2.053 \\
\mathrm{Ba}\end{array}$ & $\mathrm{R}$ & $\mathrm{R}$ & Beta & - & - \\
\hline \multirow{3}{*}{ BP3 } & $\mathrm{CBA}+$ & 52 & Long and short & St, Nd, Ci & $\mathrm{Ci}$ & + & $\begin{array}{c}1.624 \\
\mathrm{NI}\end{array}$ & $\mathrm{R}$ & $\mathrm{R}$ & Beta & - & - \\
\hline & \multirow{2}{*}{ PLET } & 85 & Short, isolated cells and in pairs & St, Nd, Ci & Ws & + & $\begin{array}{c}1.591 \\
\mathrm{NI}\end{array}$ & $\mathrm{S}$ & $\mathrm{R}$ & $\mathrm{A}$ & - & - \\
\hline & & 95 & Short, isolated cells & St, Nd, Ci & $\mathrm{Ci}$ & - & $\begin{array}{c}1.853 \\
\mathrm{Pt}\end{array}$ & $\mathrm{R}$ & $\mathrm{R}$ & A & - & - \\
\hline Vaccine & $\mathrm{CBA}$ & $\mathrm{PC}$ & $\begin{array}{c}\text { Short, isolated cells suggesting } \\
\text { a right-angle pole }\end{array}$ & St, Nd, Ci & $\mathrm{Ci}$ & - & $\begin{array}{l}2.4 \\
\mathrm{Ba}\end{array}$ & S & S & A & + & - \\
\hline $\mathrm{BC}$ & CBA & $\mathrm{NC}$ & Long & $\mathrm{C}, \mathrm{Nd}, \mathrm{Ci}$ & $\mathrm{Ci}$ & + & $\begin{array}{c}2.301 \\
\mathrm{BC}\end{array}$ & $\mathrm{R}$ & $\mathrm{R}$ & Beta & - & - \\
\hline
\end{tabular}

$a$ : higher score obtained when comparing the mass spectrum of the sample with the microbial species presented. A: absence; $\mathrm{Ba}$ : Bacillus anthracis; Bc: B. cereus; $\mathrm{Bl}$ : B. licheniformis; Bm: B. marisflavi; BP1: sample from where bovine was buried; BP2: sample from the place where the bovine was left after death (24 h); BP3: grass sample; Bs: B simplex; C: central; CA: (corral A' soil ; CB: (corral B' soil); CBA: Columbia agar base added 5\% defibrinated ram blood; CBA+: Columbia agar base added 5\% defibrinated sheep blood (0.25 M sodium acetate); CBA++: Columbia agar base added \% sheep defibrinated blood with $0.5 \%$ sodium bicarbonate; Ci: cylindrical; CT: [Army Technology Center (CTEx) soil]; E: elliptical; Long: have more than six cells; $\mathrm{NA}$ : nutrient agar medium without containing $\mathrm{NaCl}$; NA+: nutrient agar medium without containing $\mathrm{NaCl}$ and $0.25 \mathrm{M}$ sodium acetate; NC: negative control; Nd: no deformans; PC: positive control; PLET: agar (Sigma-Aldrich - 15 g/L agar, 30,000 U/L polymyxin b, 300,000 U/L lysozyme, $300 \mathrm{mg} / \mathrm{L}$ EDTA and $40 \mathrm{mg} / \mathrm{L}$ thallium acetate); Pt: Paenibacillus thiaminolyticus; R: resistant; S: susceptibility; SD: slightly deforming; Se: without sporangia; Short: have up to sex cells; St: subterminal; Ws: without spore.

Results for $\mathrm{Ca}^{2+}$ analysis showed that, according to the parameters suggested by EMBRAPA, ${ }^{(15)} \mathrm{BP} 1, \mathrm{CA}$ and $\mathrm{CB}$ presented high $\mathrm{Ca}^{2}+$ content $(>3 \mathrm{cmol} / \mathrm{dm} 3)$, $\mathrm{CT}$, medium $\mathrm{Ca}^{2+}$ content $(1-3 \mathrm{cmol} / \mathrm{dm} 3)$ and $\mathrm{BP} 2$ and $\mathrm{BP} 3$ presented low $\mathrm{Ca}^{2+}$ content $(<1 \mathrm{cmol} / \mathrm{dm} 3)$.

Regarding organic matter content, samples BP1, $\mathrm{CB}$ and CT presented high levels $(>3.0 \mathrm{dag} / \mathrm{kg}$ or $>$ $30.0 \mathrm{~g} / \mathrm{dm} 3)$, while, BP2, BP3 and CA presented average levels (1.5-3.0 dag/kg or 15-30 g/dm3), according to EMBRAPA. ${ }^{(15)}$

Isolation and identification of B. anthracis suspected bacteria - Bacterial growth was observed after incubation in aerobiosis. After observation of macroscopic characteristics, 369 colonies were Gram stained and 52 isolates were selected based on morphological and staining characteristics, as shown in Table IV.
Phenotypic and genomic identification of B. anthracis suspect isolates - From 52 isolates, 16 were negative for motility test, one strain showed susceptibility for gamma phage and penicillin and 10 were gamma phagesusceptible and penicillin-resistant. MADI-TOF/MS identified one isolate as $B$. anthracis (score above 2.3) and 11 isolates as belonging to the genus Bacillus. Two strains, 29 and 41, showed amplification of pag gene, pag amplicons from isolates 29 and 41 showed 100\% and $99.6 \%$ identity to $\mathrm{pXO1}$, respectively.

Isolate 29 was identified as $B$. anthracis (not motile, PCR positive for pag, non-haemolytic, gamma phage/ penicillin-susceptible and MALDI-TOF score 2.315) and isolate 41 was not identified as $B$. anthracis, but was placed in B. cereus group (motile, PCR positive for pag, haemolytic, gamma phage and penicillin-resistant and 
TABLE III

Physicochemical characteristics of soil samples

\begin{tabular}{|c|c|c|c|c|c|c|}
\hline Properties & $\mathrm{BP} 1^{a}$ & $\mathrm{BP} 2^{a}$ & $\mathrm{BP} 3^{a}$ & $\mathrm{CA}^{a}$ & $\mathrm{CB}^{a}$ & $\mathrm{CT}^{a}$ \\
\hline $\begin{array}{l}\text { Granulometry } \\
\mathrm{g} / \mathrm{kg}\end{array}$ & $\begin{array}{l}\text { Sand } 459 \mathrm{~g}, \\
\text { Silt } 297 \mathrm{~g}, \\
\text { Clay } 244 \mathrm{~g}\end{array}$ & $\begin{array}{l}\text { Sand } 506 \mathrm{~g}, \\
\text { Silt } 301 \mathrm{~g}, \\
\text { Clay } 193 \mathrm{~g}\end{array}$ & $\begin{array}{l}\text { Sand } 470 \mathrm{~g}, \\
\text { Silt } 229 \mathrm{~g}, \\
\text { Clay } 301 \mathrm{~g}\end{array}$ & $\begin{array}{l}\text { Sand } 390 \mathrm{~g}, \\
\text { Silt } 235 \mathrm{~g}, \\
\text { Clay } 375 \mathrm{~g}\end{array}$ & $\begin{array}{l}\text { Sand } 637 \mathrm{~g}, \\
\text { Silt } 196 \mathrm{~g}, \\
\text { Clay } 167 \mathrm{~g}\end{array}$ & $\begin{array}{l}\text { Sand } 778 \mathrm{~g}, \\
\text { Silt } 156 \mathrm{~g}, \\
\text { Clay } 66 \mathrm{~g}\end{array}$ \\
\hline $\mathrm{pH}$ & $\begin{array}{c}6,8 \\
\text { weak acidity }\end{array}$ & $\begin{array}{c}5,2 \\
\text { average acidity }\end{array}$ & $\begin{array}{c}5,1 \\
\text { average acidity }\end{array}$ & $\begin{array}{c}5,7 \\
\text { average acidity }\end{array}$ & $\begin{array}{c}5,8 \\
\text { average acidity }\end{array}$ & $\begin{array}{c}5,9 \\
\text { average acidity }\end{array}$ \\
\hline $\begin{array}{l}\mathrm{Ca}^{2+} \\
\mathrm{cmol} / \mathrm{dm}^{3}\end{array}$ & $\begin{array}{c}6,7 \\
\text { high content }\end{array}$ & $\begin{array}{c}0,7 \\
\text { low content }\end{array}$ & $\begin{array}{c}0,3 \\
\text { low content }\end{array}$ & $\begin{array}{c}4,7 \\
\text { high content }\end{array}$ & $\begin{array}{c}8,8 \\
\text { high content }\end{array}$ & $\begin{array}{c}2,8 \\
\text { average content }\end{array}$ \\
\hline $\begin{array}{l}\text { Organic matter } \\
\mathrm{g} / \mathrm{dm}^{3}\end{array}$ & $\begin{array}{c}\quad 68,3 \\
\text { high content }\end{array}$ & $\begin{array}{c}25,2 \\
\text { average content }\end{array}$ & $\begin{array}{c}25,0 \\
\text { average content }\end{array}$ & $\begin{array}{c}25,5 \\
\text { average content }\end{array}$ & $\begin{array}{c}30,3 \\
\text { high } \\
\text { content }\end{array}$ & $\begin{array}{c}34,3 \\
\text { high content }\end{array}$ \\
\hline Textural class & Loam & Loam & Sandy clay loam & Clay loam & Sandy loam & Loamy sand \\
\hline
\end{tabular}

$a$ : BP1 - cattle burier site. BP2: site where the bovine was left after death for $24 \mathrm{~h}$; BP3: grass sample; CA: corral A soil sample; CB: corral B soil sample; CT: Army Technology Center (CTEx) soil sample.

\section{TABLE IV}

Number of colonies selected according to macroscopic and microscopic characteristics and in each culture media

\begin{tabular}{|c|c|c|c|c|c|c|c|c|c|c|c|c|c|c|}
\hline \multirow[b]{3}{*}{ Samples } & \multicolumn{12}{|c|}{ Culture medium } & & \\
\hline & \multicolumn{2}{|c|}{ NA } & \multicolumn{2}{|c|}{$\mathrm{NA}+$} & \multicolumn{2}{|c|}{ CBA } & \multicolumn{2}{|c|}{ CBA+ } & \multicolumn{2}{|c|}{$\mathrm{CBA}++$} & \multicolumn{2}{|c|}{ PLET } & \multicolumn{2}{|c|}{ Total } \\
\hline & Mac & Mic & Mac & Mic & Mac & Mic & Mac & Mic & Mac & Mic & Mac & Mic & Mac & Mic \\
\hline BP1 soil & 12 & 0 & 10 & 0 & 12 & 1 & 9 & 3 & 9 & 0 & 9 & 8 & 61 & 12 \\
\hline BP2 soil & 13 & 0 & 12 & 0 & 10 & 0 & 8 & 0 & 9 & 0 & 4 & 4 & 56 & 4 \\
\hline BP3 soil & 10 & 0 & 10 & 0 & 12 & 0 & 9 & 1 & 8 & 0 & 10 & 2 & 59 & 3 \\
\hline CT soil & 14 & 4 & 10 & 2 & 13 & 3 & 11 & 3 & 10 & 1 & 12 & 8 & 70 & 21 \\
\hline CA soil & 13 & 4 & 12 & 0 & 10 & 0 & 12 & 0 & 10 & 0 & 10 & 7 & 67 & 11 \\
\hline CB soil & 11 & 0 & 9 & 1 & 13 & 0 & 10 & 0 & 9 & 0 & 4 & 0 & 56 & 1 \\
\hline Total & 73 & 8 & 63 & 3 & 70 & 4 & 59 & 7 & 55 & 1 & 49 & 29 & 369 & 52 \\
\hline
\end{tabular}

$\mathrm{NA}$ : nutrient agar without $\mathrm{NaCl}$; $\mathrm{NA}+$ : nutrient agar with $0.25 \mathrm{M}$ sodium acetate; $\mathrm{CBA}$ : Columbia agar base added $5 \%$ defibrinated sheep blood; $\mathrm{CBA}+$ : Columbia agar base added $5 \%$ defibrinated sheep blood with $0.25 \mathrm{M}$ sodium acetate; CBA++: Columbia base agar added 5\% sheep defibrinated blood with $0.5 \%$ sodium bicarbonate; PLET: agar polymyxin B, lysozyme, EDTA and thallium acetate; Mac: macroscopic; Mic: microscopic.

MALDI-F score 1.849) and was considered as $B$. anthracis-like bacterium.16S rRNA gene sequencing identified isolate 29 as belonging to $B$. cereus group showing $100 \%$ of identity and query cover with $B$. anthracis ATCC141576 (GenBank accession MT994366), while isolate 41 presented $100 \%$ identity and $94 \%$ query cover with eight species belonging to the $B$. cereus group (GenBank accession MT994363 - Bacillus cereus strain IAM 12605, B. pacificus strain MCCC 1A06182, B. paranthracis strain MCCC 1A00395, B. cereus strain CCM 2010, B. cereus strain NBRC 15305, B. cereus ATCC 14579 (16S RNA mA), Bacillus cereus ATCC 14579 and $B$. cereus strain JCM 2152). In addition, isolates 2, 3, 4, $6,7,8,30,36$ and 37 (all gamma phage-susceptible and penicillin resistant, $\mathrm{pXO1-}$ and $\mathrm{pXO} 2-)$ were identified as belonging to the $B$. cereus group (GenBank accessions: MT994549, MT993863, MT994256, MT993936, MT993895, MT994361, MT994451, MT994163 and MT993931, respectively) (Table II and Table V).

\section{DISCUSSION}

In this study, one $B$. anthracis strain (harbouring $\mathrm{pXO1}$, but not $\mathrm{pXO} 2$ ), one strain of $B$. cereus group harboured a pXO1-like plasmid (99.6\% similarity with pag) and 10 Bacillus strains were isolated (Table V) from different soils of Brazil. These 10 isolates might be $B$. anthracis strains lacking virulence plasmids, which is in agreement with the findings of Kolton et al. ${ }^{(22)}$ The probability of positive results for $B$. anthracis species cannot be ruled out, as there are reports of such occur- 
TABLE V

Results of phenotypic and molecular identification tests of 52 isolates

\begin{tabular}{|c|c|c|}
\hline Tests of isolates & & Number of isolates in each test \\
\hline \multirow{2}{*}{ Motility test } & Non-motile & 36 \\
\hline & Motile & 16 \\
\hline \multirow{4}{*}{ MALDI-TOF } & Score above 2.3 & 1 identified as Bacillus anthracis \\
\hline & Score values between $2.0-2.3$ & 11 confirmed as Bacillus sp. \\
\hline & Score values between $1.7-2.0$ & 22 identified as probable Bacillus sp. \\
\hline & Score values lower than 1.7 & 18 were not identified \\
\hline \multirow{4}{*}{$\begin{array}{l}\text { Susceptibility } \\
\text { to gamma } \\
\text { phage and } \\
\text { penicillin }\end{array}$} & Susceptible to gamma phage and penicillin & 1 \\
\hline & Susceptible to gamma phage and resistant to penicillin & 10 \\
\hline & Resistant to gamma phage and penicillin & 13 \\
\hline & Resistant to gamma phage and susceptible to penicillin & 28 \\
\hline \multirow{2}{*}{ PCR } & $\mathrm{pXO} 1+/ \mathrm{pXO} 2-$ & 02 \\
\hline & pXO1-/pXO2- & 50 \\
\hline \multirow{2}{*}{$\begin{array}{l}\text { Amplicon } \\
\text { sequencing }\end{array}$} & \multirow{2}{*}{ Identity to pag (surogate of pXO1) } & Isolate 29 (100\% identity) \\
\hline & & Isolate $41(99.6 \%$ identity $)$ \\
\hline \multirow{3}{*}{ 16sRNA } & \multirow{3}{*}{ Identified as strain belonging to B. cereus group } & $\begin{array}{l}\text { Isolate } 29 \text { (100\% identity and } 100 \% \text { query cover to } \\
\text { B. paramycoides strain MCCC 1A04098, B. albus } \\
\text { strain MCCC } 1 \text { A02146 and B. anthracis ATCC141576 }\end{array}$ \\
\hline & & $\begin{array}{l}\text { Isolate } 41 \text { (100\% identity and } 94 \% \text { query cover to } \\
\text { eight species belonging to the } B . \text { cereus group) }\end{array}$ \\
\hline & & $\begin{array}{c}\text { Isolates } 2,3,4,6,7,8,30,36 \text { and } 37 \text { (match to several } \\
\text { species belonging to the } B \text {. cereus group) }\end{array}$ \\
\hline
\end{tabular}

rence in the literature. ${ }^{(23,24)}$ These isolates were recovered from six different culture media. From PLET cultures, a pXO1+ B. anthracis was isolated, from NA, a pXO1+ B. anthracis-like was isolated. These data corroborated what is described in literature, which suggest PLET is the most selective medium for $B$. anthracis isolation. ${ }^{(6)}$

This study is the first on this subject performed in Brazil. It showed the importance of active surveillance in soil and the correct identification of the isolates for better understanding $B$. anthracis distribution in nature and for the elucidation of possible outbreaks in Brazil.

Several methods for isolation and identification $B$. anthracis in soil have been reported, although there is no consensus among the respective studies. A direct method was chosen, because it is a simple and economical protocol for survey B. anthracis in soil samples. Among the soils analysed in this study, the sample presenting characteristics that would favour the prevalence of $B$. anthracis spores was BP1, in according to Shadomy et al. ${ }^{(25)}$ Interestingly, neither $B$. anthracis nor B. anthracislike strains were isolated from this sample. In the USA, the bacterium was isolated from soils with neutral to alkaline $\mathrm{pH}$ and high concentrations of nitrogen, while in Germany, there was no correlation with the type of soil. These differences indicate that the occurrence depends not only on the type of soil, but also on environmental conditions, the pathogen's life cycle, persistence, ecolo- gy and the ability to lose or acquire virulence attributes. ${ }^{(6,26)} \mathrm{B}$. anthracis (harbouring $\mathrm{pXO}$, but not $\mathrm{pXO} 2$, strain 29) was isolated from the CA soil, a clay loam, with medium acidity, high $\mathrm{Ca}^{2+}$ and medium organic matter content. B. anthracis was isolated in the former USSR from soils with medium acidity. ${ }^{(26)}$ An isolate (strain 41) from CT soil cultured in NA presented microscopic characteristics of large Bacillus was resistant to penicillin and gamma phage, motile and beta haemolytic and would not be regarded as $B$. anthracis. However, this isolate harboured a pXO1-like plasmid $(99.6 \%$ similarity with pag) and might be a $B$. anthracis-like bacterium. It was described that $7 \%$ of $1,000 \mathrm{~B}$. cereus sensu lato isolated from environmental samples (soil, water, insects, plants) were shown to contain pXO1-like and/or pXO2like plasmids. B. mycoides harbouring pXO1-like and/or pXO2-like plasmids is less related than B. cereus or $B$. thuringiensis. It is relevant to note that $B$. anthracis-like strains have already been described causing disease with the same symptoms as anthrax in animals and humans, including being lethal. This suggested the occurrence of plasmid transfer events across the different species of the $B$. cereus group. ${ }^{(27)}$

Species allocated in the B. cereus group have high genetic identity, which makes the differentiation complex, even by means of molecular tests. ${ }^{(28,29)}$ In this group of bacteria, species classification is still based on pheno- 
typic and genotypic traits, pathogenicity, host preference and ecological niche. ${ }^{(23)}$ Phenotypic tests are essential for $B$. anthracis identification. However, those considered to be classic phenotypic characteristics may not be present in all B. anthracis strains. Therefore, Kovac et al. ${ }^{(30)}$ proposed phylogenetic classification based on the complete genome sequencing for bacteria belonging to the $B$. cereus group. The isolation of 10 strains presenting all the phenotypic characteristics of the specie, but not harbouring $\mathrm{pXO} 1$ neither $\mathrm{pXO} 2$ corroborated what were described in the literature.

Molecular assay in MALDI-TOF MS analysis, the only strain with a score above 2.3 , which confirms species identification, was the one isolated from CA soil (strain 29). This isolate was confirmed as B. anthracis by other methods applied in this study. Among the isolates analysed, several were identified only at genus level, while others were identified as non-Bacillus, although the results of physiological tests had identified them as Bacillus spp.

Due to the inherent difficulty in differentiating between species with such a genomic similarity, it is necessary to enlarge the number of spectra in MALDITOF library in order to achieve better results for $B$. anthracis and $B$. anthracis-like bacteria identification. ${ }^{(30)}$ Although PCR has the potential to infer the presence pXO1 and pXO2 plasmids, the method does not inform the species and the lineage unequivocally. Total genome sequencing has been shown to be the best methodology to study geographical distribution of strains during natural outbreaks or events suspected of bioterrorism. . $^{(2,24)}$ 16sRNA sequencing showed that it is difficult to differentiate strains that belong to the $B$. cereus group. These results are due to the remarkable $99.6 \%$ identity percentage what makes them indistinguishable from each other when 16sRNA gene subunit sequencing was made. $(23,24,29)$ These results showed the importance of whole genome sequencing for identification of bacteria belonging to the $B$. cereus group.

To date, the literature describes that virulent strains of $B$. anthracis necessarily harbour both plasmids. However, strains of $B$. cereus harbouring plasmid-like may cause fatal inhalation infection similar to inhalation anthrax, and cases in humans have already been described. Due to Brazil's continental dimensions, a large diversity of soils and climates can be found. The samples analysed represent only a fraction of this diversity. Nevertheless, it is important to highlight that this work showed promising results and it was the first study to report results from an active surveillance for B. anthracis in Brazil.

In conclusion, since livestock is one of the main economic activities in Brazil, large-scale studies involving B. anthracis active surveillance in soil in Brazil should be performed to reduce and/or prevent economic losses, and a complete characterisation of the isolates should be carried out in order to provide accurate information about Brazilian strains. In this work, we isolated $B$. anthracis ( $\mathrm{pXO1}^{+}$and $\mathrm{pXO} 2-$ ) and $B$. anthracis-like in soil samples, being the first description of this type in Brazil. This data increases the need to carry out surveillance of these species in the soil. The correct identification of these species is of paramount importance for the knowledge of the pathogen distribution in nature and elucidation of possible outbreaks.

\section{ACKNOWLEDGEMENTS}

To Col Marcos Barcellos, Director of IDQBRN/EB, Dr Marcelo M Werneck, of the laboratory of LIF/COPPE/UFRJ, Dr Leonardo Rusak, of the laboratory of LFB/Fiocruz, DVM Paula Amorim and DVM Luis Armando Calvão Brust, of DSA-RJ/MAPA, and Dr Bassam Hallis, of the laboratory of HPA/PHE, for had supported this study. The authors have declared no conflicts of interest.

\section{AUTHOR'S CONTRIBUTION}

AMV and LR developed the research methodology. JRSS performed all collections and laboratory analyses, in addition to writing the work together with AMV and LR. MFSG, RCSBA, MMW, RBR, RCP and FBOL contributed to the implementation of the research, to the analysis of the results and to the writing of the manuscript.

\section{REFERENCES}

1. Ehling-Schulz M, Lereclus D, Koehler TM. The Bacillus cereus group: Bacillus species with pathogenic potential. Microbiol Spectr. 2019;7(3):1-60. Available from: https://www.ncbi.nlm.nih. gov/pmc/articles/PMC6530592/

2. Moayeri M, Leppla SH, Vrentas C, Pomerantsev AP, Liu S. Anthrax pathogenesis. Annu Rev Microbiol. 2015;69(1):185-208. Available from: https://www.researchgate.net/publication/280241634_Anthrax_Pathogenesis

3. Sweijs T, Sinning P, Bontje E, Frattina C, Frattina D, Abdalla $\mathrm{M}$. The increasing threat of biological weapons handle with sufficient and proportionate care. HCSS. 2016:1-38. Available from: https://hcss.nl/sites/default/files/files/reports/Threat $\% 20$ and $\% 20$ Care\%20of\%20BWdef4eversie_0.pdf

4. Nuclear Threat Initiative. Russia: Biological. 2015. Available from: https://www.nti.org/learn/countries/russia/biological/

5. Ravenel MP. Anthrax in man and animals. Am J Public Heal Nations Heal. 2008;30(3):299-300. Available from: https://www.who. int/csr/resources/publications/anthrax_webs.pdf

6. Silvestri E, Griffin DW. Processing protocol for soil samples potentially contaminated with Bacillus anthracis spores. EPA. 2017;(2):1-28. Available from: https://cfpub.epa.gov/si/si_public_ record_report.cfm?Lab $=$ NHSRC $\&$ dirEntryId $=335822$

7. Carlson CJ, Getz WM, Kausrud KL, Cizauskas CA, Blackburn JK, Carrillo FAB, et al. Spores and soil from six sides: interdisciplinarity and the environmental biology of anthrax (Bacillus anthracis). Biol Rev. 2018;93(4):1813-31.

8. Schild AL, Sallis ES V, Soares MP, Ladeira SR.L, Schramm R, Priebe AP, et al. Anthrax in cattle in southern Brazil: 1978-2006. Pesqui Vet Bras. 2006;26(4):243-8. Available from: https://www. scielo.br/pdf/pvb/v26n4/a09v26n4.pdf

9. World Organisation for Animal Health. WAHIS Interface. Zoonotic diseases in Humans. Available from: w.w.w.oie.int/wahis_2/ public/wahid.php/Countryinformation/Zoonoses

10. Ministério da Agricultura, Pecuária e Abastecimento (BR). Coordenação de Informação e Epidemiologia - Saúde Animal. 2018. Available from: http://indicadores.agricultura.gov.br/saudeanimal/index.htm

11. Primio R. Nove casos de carbúnculo hemático. Arq Rio Gd Med. 1932:452-470. Available from: http://webcache.googleusercontent.com/search?q=cache:Yq0ixwrlUBEJ:seer.ufrgs.br/riograndemed/article/download/32005/20010+\&cd=1\&hl=en\&ct=clnk\& $\mathrm{gl}=\mathrm{br} \& \mathrm{client}=$ firefox $-\mathrm{b}-\mathrm{d}$ 
12. Estima-Silva P, Molarinho KR, Marcolongo-Pereira C, Ladeira SRL, Schramm R, Priebe AP, et al. Morte súbita em bovinos no sul do Rio Grande do Sul: epidemiologia e diagnóstico. Pesqui Vet Bras. 2016;36(1):19-23. Available from: https://www.scielo.br/pdf/ $\mathrm{pvb} / \mathrm{v} 26 \mathrm{n} 4 / \mathrm{a} 09 \mathrm{v} 26 \mathrm{n} 4 . \mathrm{pdf}$

13. Mari AS, Kamboh AA, Khalid-UR-Rehman B, Sahito IA, Abro SH. Prevalence of Bacillus anthracis spores in soil of district Badin. J Anim Heal Prod. 2017;(2):1-25. Available from: http://nexusacademicpublishers.com/uploads/files/JAHP_5_2_79-84.pdf

14. Guimarães PTG, Alvarez VH, Ribeiro AC. Recomendações para o uso de corretivos e fertilizantes em Minas Gerais $-5^{\text {a }}$ Aproximação. Comissão de Fertilidade do Solo do Estado de Minas Gerais (CFSEMG). 1999:369. Available from: http://ead.senar.org.br/lms/ webroot/uploads/senar/duvidas/res_110155

15. Sobral LF, Barreto MC V, Silva AJ da, Anjos JL dos. Guia prático para interpretação de resultados de análises de solo. Embrapa Tabuleiros Costeiros - Documentos. INFOTECA-E. 2015:13. Available from: https://www.infoteca.cnptia.embrapa.br/infoteca/ bitstream/doc/1042994/1/Doc206.pdf

16. García-Gaines RA, Frankenstein S. USCS and the USDA Soil Classification System. Development of a Mapping Scheme. UPRM ERDC Educ Res Internsh Progr. 2015;(3):1-43. Available from: https://apps.dtic.mil/dtic/tr/fulltext/u2/a614144.pdf

17. Lasch P, Beyer W, Nattermann H, Stammler M, Siegbrecht E, Grunow R, et al. Identification of Bacillus anthracis by using matrix-assisted laser desorption ionization-time of flight mass spectrometry and artificial neural networks. Appl Environ Microbiol. 2009;75(22):7229-42. Available from: https://www.ncbi.nlm.nih. gov/pmc/articles/PMC2786504/pdf/0857-09.pdf

18. Beyer W, Glöckner P, Otto J, Böhm R. A nested PCR method for the detection of Bacillus anthracis in environmental samples collected from former tannery sites. Microbiol Res. 1995;150(2):179-86.

19. World Organisation for Animal Health. Manual of diagnostic tests and vaccines for terrestrial animals - Anthrax. Terrestrial Manual. 2019;(8):1-1833. Available from: https://www.oie.int/standardsetting/terrestrial-manual/

20. Health Protection Agency England. Standard operating procedure: further characterization of PCR positive Bacillus anthracis isolates -SPATH-065/02-15. 2015;1-4.

21. Watts JL, Lowery DE, Teel JF, Ditto C, Horng JS, Rossbach S. Phylogenetic studies on Corynebacterium bovis isolated from bovine mammary glands. J Dairy Sci. 2001;84(11):2419-23. Available from: https://www.journalofdairyscience.org/article/S00220302(01)74691-7/pdf
22. Kolton CB, Podnecky NL, Shadomy S V., Gee JE, Hoffmaster AR. Bacillus anthracis gamma phage lysis among soil bacteria: an update on test specificity. BMC Res Notes. 2017;10(1):4-9. Available from: https://bmcresnotes.biomedcentral.com/track/ pdf/10.1186/s13104-017-2919-8

23. Liu Y, Lai Q, Göker M, Kolthoff JPM, Wang Meng, Sun Y, et al. Genomic insights into the taxonomic status of the Bacillus cereus group. Sci Rep. 2015;5:1-11. Available from: https://www.nature. com/articles/srep14082.pdf

24. Valseth K, Nesbø CL, Easterday WR, Turner WC, Olsen JS, Stenseth NC, et al. Temporal dynamics in microbial soil communities at anthrax carcass sites. BMC Microbiol. 2017;17(1):1-15. Available from: https://bmcmicrobiol.biomedcentral.com/track/pdf/10.1186/ s12866-017-1111-6

25. Food and Agriculture Organization of the United Nations. Anthrax outbreaks: a warning for improved prevention, control and heightened awareness. Empres Watch. 2016;37(9):8. Available from: http://www.fao.org/3/a-i6124e.pdf

26. Mitscherlich E, Marth EH. Microbial survival in the environment: bacteria and Rickettsiae important in human and animal health. New York (NT): Springer Verlag; 1984.

27. Marston CK, Ibrahim H, Lee P, Churchwell G, Gumke M, Stanek $\mathrm{D}$, et al. Anthrax toxin-expressing bacillus cereus isolated from an anthrax-like eschar. PLoS One. 2016;11(6):1-7. Available from: https://www.ncbi.nlm.nih.gov/pmc/articles/PMC4892579/pdf/ pone.0156987.pdf

28. Hu X, Swiecicka I, Timmery S, Mahillon J. Sympatric soil communities of Bacillus cereus sensu lato: population structure and potential plasmid dynamics of pXO1- and pXO2-like elements. FEMS Microbiol Ecol. 2009;70(3):344-55. Available from: https:// academic.oup.com/femsec/article/70/3/344/530754

29. Zwick ME, Joseph SJ, Didelot X, Chen PE, Bishop-Lilly KA, Stewart AC, et al. Genomic characterization of the Bacillus cereus sensu lato species: Backdrop to the evolution of Bacillus anthracis. Genome Res. 2012;22(8):1512-24. Available from: https:// www.ncbi.nlm.nih.gov/pmc/articles/PMC3409264/

30. Kovac J, Miller RA, Carroll LM, Kent DJ, Jian J, Beno MS, et al. Production of hemolysin BL by Bacillus cereus group isolates of dairy origin is associated with whole-genome phylogenetic clade. BMC Genomics. 2016;17(1):1-16. Available from: https://bmegenomics.biomedcentral.com/track/pdf/10.1186/s12864-016-2883-z 